\title{
Thermo-Kinetic Anomalies across Rigidity Threshold in $\mathrm{Ge}_{x} \mathrm{Se}_{1-x}$
}

\author{
Anand M. Awasthi ${ }^{1, * 1}$ and Sujatha Sampath ${ }^{2, * 2}$ \\ ${ }^{1}$ Inter University Consortium for DAE Facilities University Campus, Khandwa Road, Indore-452 017, India \\ ${ }^{2}$ IPNS Division, Argonne National Laboratory 9700, S. Cass. Ave., Argonne, IL-60439, USA
}

We have investigated the glass-transition kinetics of nine $\mathrm{Ge}_{x} \mathrm{Se}_{1-x}$ glasses by differential scanning calorimetry. Relation between the drive (heating-rate $q$ ) and response (heatflow shift $\Delta H$ at $T_{\mathrm{g}}$ ) is seen to be strictly linear only for GeSe 4 , known to signify the bulk-rigidity threshold for this series. From an Arrhenius analysis the activation energies for glassy relaxation are estimated, and point to the existence of different thermokinetic phases below and above the threshold composition. Series behaviour of the kinetic activation is conciled to a concurring one seen in the size of cooperatively diffusing regions. The anomalies are attributed to structural crossovers with Ge doping; first from the parent uniform Se-chains to that of backbones out-branching at corner-shared $\mathrm{Ge}\left(\mathrm{Se}_{1 / 2}\right)_{4}$ tetrahedral clusters, and subsequently interconnecting by edge-shared configurations to realize a random pearl-necklace 3-D covalent network.

(Received Feburary 18, 2002; Accepted March 12, 2002)

Keywords: rigidity percolation, glasses, differential scanning calorimetry.

\section{Introduction}

Chalcogenide glasses make interesting study because they evolve into continuous random network (CRN) structure ${ }^{1)}$ by appropriate doping. It was Phillips who first introduced the notion of highest glass-forming propensity in covalent networks ${ }^{2,3)}$ near a mean atomic coordination of 2.4. Concurrently, Thorpe conceptualized the floppy \& rigid vibration modes ${ }^{4)}$ and the occurrence of a 'rigidity threshold' in CRN systems. Angell's subsequent classification of kinetically fragile/strong glass-formers ${ }^{5)}$ is in conformity with these ideas. In the binary system $\mathrm{Ge}_{x} \mathrm{Se}_{1-x}$, the elemental polymeric-Se is cross-linked with the increasing Ge admixture nucleating $\mathrm{Ge}\left(\mathrm{Se}_{1 / 2}\right)_{4}$ tetrahedra, that coalesce first into corner-shared clusters branching out the backbone structural units, the latter in turn gets networked via the edge-shared configurations. At the doping fraction $x_{\mathrm{p}}=0.2$ the mean coordination $\langle r\rangle$ of $2.4(=2 x+2)$ is realized in $\mathrm{GeSe}_{4}{ }^{6}{ }^{6}$

In the glass-transition studies using DSC, ${ }^{7-9)}$ though elaborate thermal procedures are employed and their effects on the transition characteristics reported, an examination of the drive-response relationship is missing. Moreover, relatively scarce studies ${ }^{10)}$ relate these characteristics to appropriate physical parameters. The fact that temperature-scanning experiments are essentially of non-equilibrium kind ${ }^{11)}$ has to be taken into account while interpreting its thermogram. Examining glass transition at various heating rates, of materials covering a breadth of property-values is one way to explore these issues.

To look at the fine effects of rigidity percolation in caloric properties of $\mathrm{Ge}_{x} \mathrm{Se}_{1-x}$, a DSC study on its three close compositions across $x=0.2$, and six flanking ones away from the same was performed. Heat flow shift $\Delta H$ across $T_{\mathrm{g}}$ was examined for its linearity vs. the scan rate $q$. By fitting Arrhenius dependence to $1 / T_{\mathrm{g}}$ vs. $\operatorname{Ln}[q]$, activation energy

\footnotetext{
${ }^{* 1}$ Corresponding author: E-mail: amawasthi@iucindore.ernet.in

${ }^{* 2}$ Formely at Inter University Consortium for DAE Facilities, University Campus, Khandwa Road, Indore-452 017, India. Present Address: IPNS Division, Argonne National Laboratory 9700, S. Cass. Ave., Argonne, IL-60439, USA. E-mail:ssampath@anl.gov
}

$E_{\mathrm{a}}$ was evaluated for all compositions, and interpreted in the context of the co-diffusing regions CRR. ${ }^{12)}$ Anomalous compositional dependence in these physical attributes is clearly discernible away from $\mathrm{GeSe}_{4}$.

\section{Experimental}

Bulk glasses $\mathrm{Ge}_{x} \mathrm{Se}_{1-x}$ for $x=0.15,0.16,0.175,0.19$, $0.20,0.21,0.23,0.27$, and 0.30 were prepared by standard melt-quench technique ${ }^{13)}$ from elemental $\mathrm{Ge}$ and $\mathrm{Se}(5 \mathrm{~N}$ purity, CERAC). The compositions were confirmed to $\pm 0.2 \mathrm{Ge}$ at $\%$ by inductively coupled plasma-atomic emission spectroscopy (ICP-AES) analysis. Amorphous nature of the samples was verified by XRD, which showed no crystalline Bragg peaks. Their glassy character was determined using a Differential Scanning Calorimeter (TA Instruments Model 2910). The as-quenched samples were well equilibrated at room temperature for 8 to 10 days before performing the DSC scans.

DSC scans at ramps $(q) 3,5,10,15$, and $20 \mathrm{~K} / \mathrm{min}$ were carried out to examine the heating rate systematic. The imposed rates were followed by the samples to within $1 \%$. Our $T_{\mathrm{g}}$ 's determined from the inflexion point in the $H$ vs. $T$ scans are given in Table 1, with other reported values. ${ }^{14-16)}$

Table 1 Glass transition temperatures $T_{\mathrm{g}}$ for nine $\mathrm{Ge}_{x} \mathrm{Se}_{1-x}$ compositions.

\begin{tabular}{ccccc}
\hline$x($ atGe $)$ & Present $(5 \mathrm{~K} / \mathrm{m})$ & Ref. 14) $(3 \mathrm{~K} / \mathrm{m})$ & Ref. 15) & Ref. 16) $(10 \mathrm{~K} / \mathrm{m})$ \\
\hline 15 & 398.75 & 408.71 & 382.50 & 406.33 \\
\hline 16 & 401.46 & & & \\
\hline 17.5 & 395.37 & & & \\
\hline 19 & 437.70 & 448.00 & --- & -- \\
\hline 20 & 448.56 & 462.28 & 437.50 & 456.33 \\
\hline 21 & 467.36 & 471.21 & --- & -- \\
\hline 23 & 485.20 & & & \\
\hline 27 & 512.37 & & & 623.00 \\
\hline 30 & 585.73 & 598.00 & 572.50 & \\
\hline
\end{tabular}




\section{Results and Discussion}

In Fig. 1(a) we show the heat flow signals for $\mathrm{GeSe}_{4}$ at the five heating rates, after correcting for the baseline variations. The curves have been shifted to join at low temperature and the $T_{\mathrm{g}}$ 's determined are uncertain by $\pm 0.2 \mathrm{~K}$. Recognizing the heat flow step $\Delta H$ at $T_{\mathrm{g}}$ as the material's caloric response, it is of interest to see its dependence on the applied excitation viz., the heating ramp $q$. Certainly the heat capacity $C_{\mathrm{p}}(=-H / q)$ as the intrinsic coupling parameter (in terms of a generalized susceptibility ${ }^{17,18)}$ is meaningful only for the linear response case. For this purpose the heat-flow shift is plotted vs. the heating ramp in Fig. 1(b). It is straightforward to realize a systematic behaviour in the $\mathrm{GeSe}_{4}$ data. A uniformly linear dependence is not so obvious for other compositions. For $\mathrm{GeSe}_{4}$ this transition parameter can be linearly fitted, while the non-linearity in $\Delta H(q)$ 's for $x \neq 0.2$ becomes more pronounced for higher $q$ values. Moreover, non-zero extrapolations of these signals for $q \rightarrow 0$ determine the accuracy of the $\Delta C_{\mathrm{p}}$ data $(=-\Delta H / q)$, important to account for the direct use of the values obtained.

Having witnessed an anomalous irregularity away from the threshold composition, we now examine the conformity of

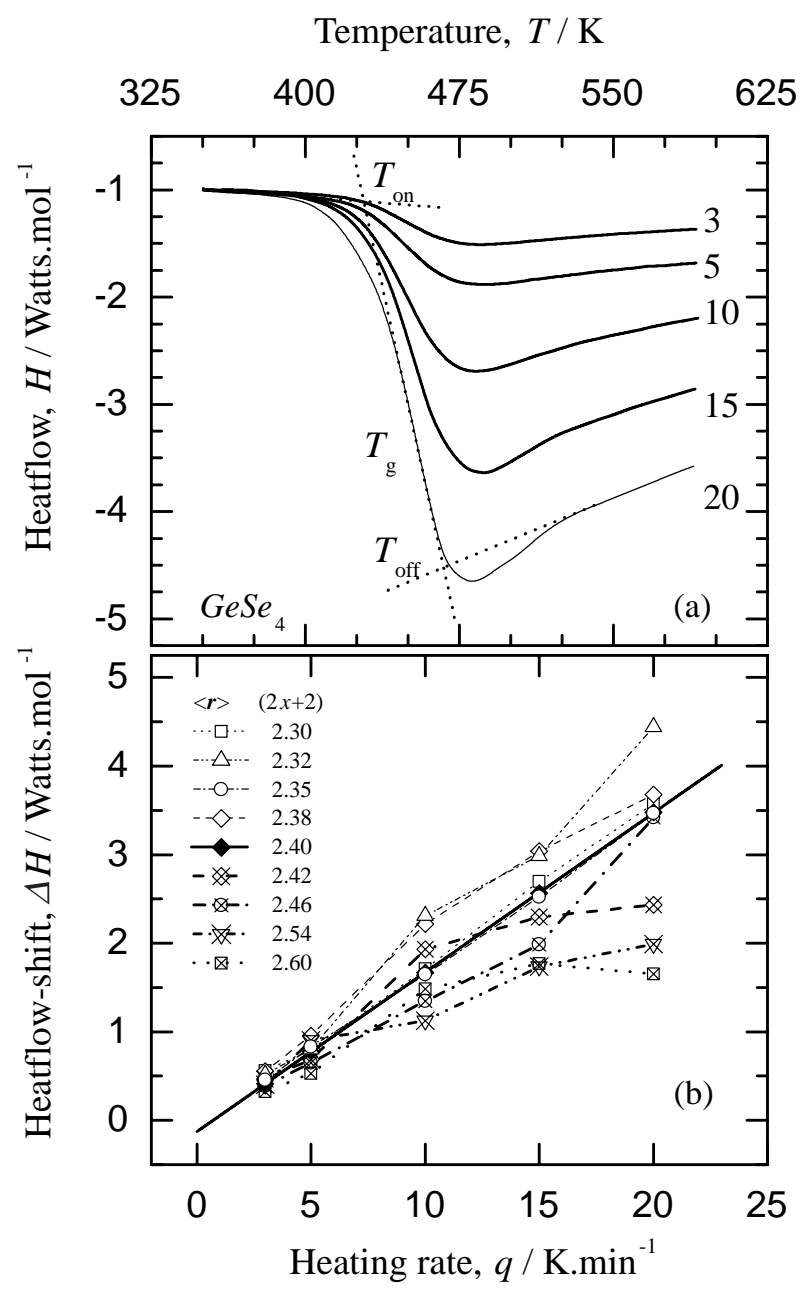

Fig. 1 (a) Caloric heat flow vs. temperature across the glass transition for $\mathrm{GeSe}_{4}$ at five heating ramps (b) heat-flow-shift at $T_{\mathrm{g}}$ vs. the heating rate $q$ for the nine $\mathrm{Ge}_{x} \mathrm{Se}_{1-x}$ compositions reported, showing remarkable linearity for $\mathrm{GeSe}_{4}$. the glass-kinetics to the standard Arrhenius formulation. The $1 / T_{\mathrm{g}}$ vs. $\operatorname{Ln}[q]$ plotted in Fig. 2(a) can be fairly fitted with straight lines, with small deviations at the higher ramps. From the slopes one determines the effective activation energies for the glass relaxation at the respective compositions, using the Arrhenius dependence $\operatorname{Ln}[q]=\mathrm{A}-E_{\mathrm{a}} / R T_{\mathrm{g}}$. The minimum and maximum variations in $T_{\mathrm{g}}$ over the entire ramprange were found to be $3 \mathrm{~K}$ and $10 \mathrm{~K}$ respectively, as shown in the close-up Fig. 2(c) and 2(d). On careful scrutiny the straight-line was found to be most optimal for the composition $x=0.2$, while it represented the farther $x$ 's to increasingly lesser degree. This observation, together with the linear response as emphasised in Fig. 1(a) indicate a saddle-pointlike regularity at $x_{\mathrm{p}}=0.2$.

The activation $E_{\mathrm{a}}$ so obtained represent the average barriers for the glasses' relaxation in their configuration space, defined by multitude of metastable energy minima. Now if the glass is tough (i.e., less fluffy and thus with fewer such minima), it undergoes this relaxation rather slowly under thermal excitation, ${ }^{19)}$ or yields poorly to deformation attempted by a mechanical force, since within it there is scarce empty space available for the same. Consequently, though the theory of Cohen and Turnbull ${ }^{20)}$ assumes no activation energy for free volume rearrangement, in glasses a distribution of hopping barriers and distances is necessitated, ${ }^{21)}$ thereby realizing higher effective energy barriers for the harder glasses. From the plot of $E_{\mathrm{a}}$ vs. the composition (atomic coordination $\langle r\rangle=2 x+2$ ) in Fig. 3(a), one can clearly delineate between the strong/fragile glass members of the $\mathrm{Ge}_{x} \mathrm{Se}_{1-x}$

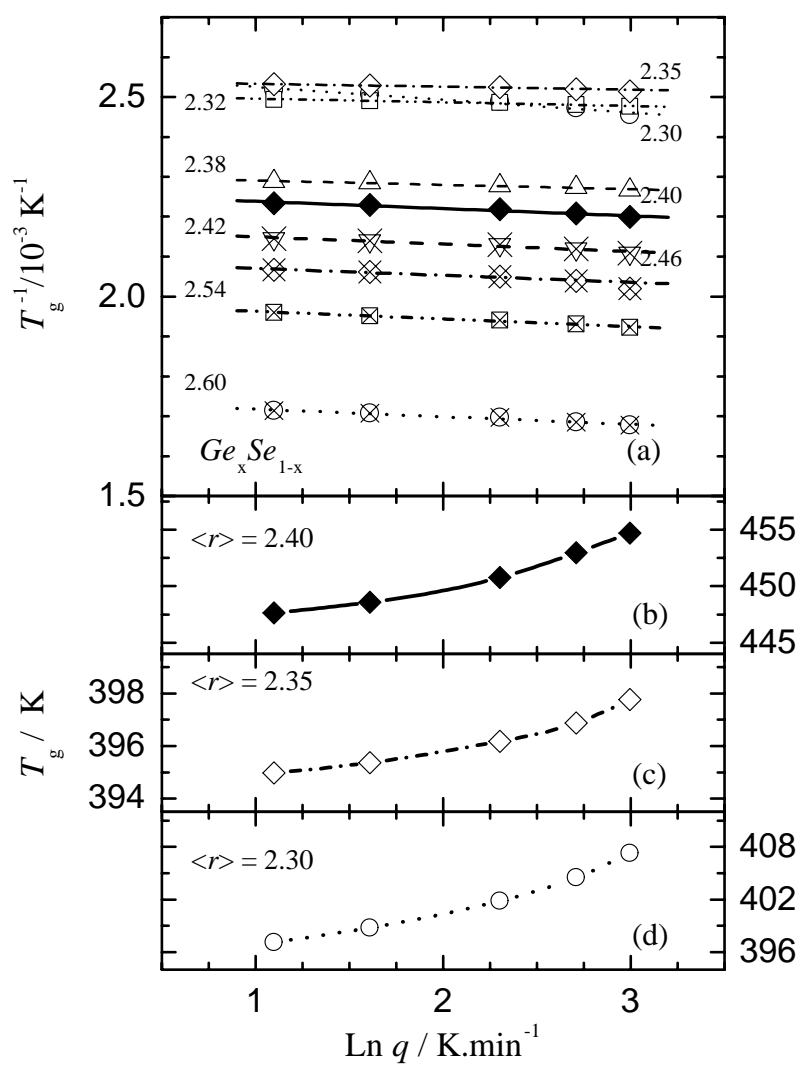

Fig. 2 (a) Arrhenius plots with fits for the glass transition kinetics. Zoomed-in illustration of typical $T_{\mathrm{g}}$ variations with ramp for (b) $x=0.20$, (c) $x=0.175$, and (d) $x=0.15$ compositions. 


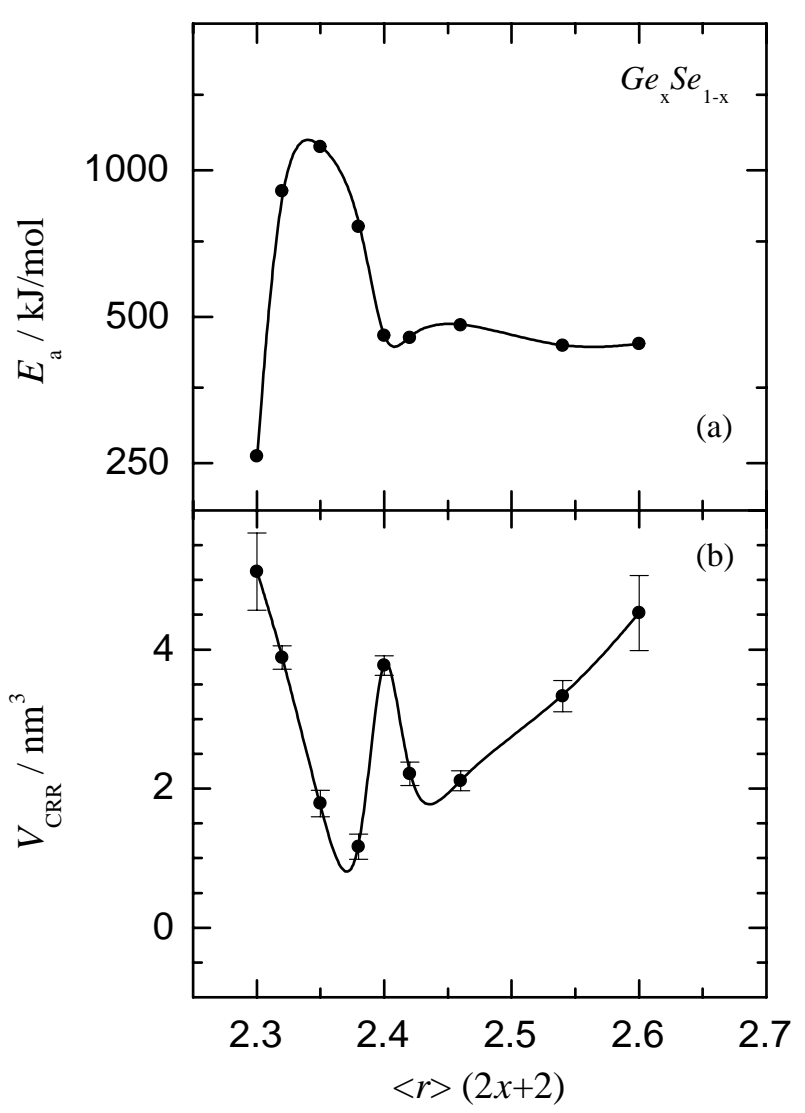

Fig. 3 (a) Activation energy $E_{\mathrm{a}}$ and (b) size of the co-diffusing region $V_{\mathrm{CRR}}$ across the $\mathrm{Ge}_{x} \mathrm{Se}_{1-x}$ series. The delineation of two different phases below and above $x_{\mathrm{p}}$ is clearly brought out.

series. Moreover, it appears that the $x<0.2$ range can be grouped to represent a thermo-kinetic block, different than the one belonging to $0.2 \leq x \leq 0.3$. An abrupt rise in characteristic energy near $\mathrm{GeSe}_{7}(\langle r\rangle=2.3)$ also indicates the emergence of a distinct 'phase'. Again the special $x_{\mathrm{p}}=0.2$ composition stands out to mark a 'phase transition'.

In order to consolidate these identifications it is worthwhile to explore the dynamic heterogeneity in the series, intertwined with the temperature-frequency scaling that develops near the glass transition. As early as 1965 Adam and Gibbs $^{11)}$ observed that as one approaches $T_{\mathrm{g}}$ from above, the fast dynamics involving fewer atoms (Johari-Goldstein $\beta$-process ${ }^{22)}$ ) decouples from the slow $\alpha$-relaxations that are characterized by cooperative motion of groups of 35-to-300 atoms. This diffusive dynamics becomes successively sluggish, as the temperature is decreased, eventually giving rise to a diverging timescale at the Vogel-Fulcher temperature $T_{0} .{ }^{23,24)}$ Such co-shifting groups of atoms define a (dynamical) cluster that rearranges its shape (and location) when it finds a void (free-volume) equal to its size available next to it. $^{20)}$

Spatial-fluctuations implying cooperatively rearranging regions $^{25)}$ define a characteristic nano-length $\zeta_{\mathrm{CRR}}$ that determines the diffusive dynamics of "particles" in the system. In general this is expected to have higher values for fragile glassformers because of their larger dynamical heterogeneity. This corresponds to larger CRRs for the soft glass, with widely distributed characteristic energy/time scales manifesting e.g., in non-Debyeian relaxation, in dielectric spectral hole burn- ing, and in multi-dimensional $\mathrm{NMR}^{26,27)}$ Assuming the CRRs in the glass to have enough number of "particles" $\left(N_{\mathrm{CRR}}\right.$, of heat capacity $c$ each) for applying thermodynamic concepts, their mean temperature fluctuation is ${ }^{28)} \delta T^{2}=k_{\mathrm{B}} T^{2} / c N_{\mathrm{CRR}}$. For $T=T_{\mathrm{g}}$ this fluctuation is given by the half-width of the glass transition interval. ${ }^{29)}$ The volume of such a subsystem of "particles" (molar mass $M$, density $\rho$ ) at $T_{\mathrm{g}}$ is then $V_{\mathrm{CRR}}=\left(M / \rho N_{\mathrm{A}}\right) N_{\mathrm{CRR}}=\left(4 k_{\mathrm{B}} M / \rho C_{\mathrm{g}}\right)\left(T_{\mathrm{g}} / \Delta T_{\mathrm{g}}\right)^{2}$.

Defining the "particle" to be one $\mathrm{Ge}_{x} \mathrm{Se}_{1-x}$ pseudo atom, the evaluated $V_{\mathrm{CRR}}$ from the data at the heating $\operatorname{ramp} q=$ $5 \mathrm{~K} / \mathrm{min}$ are plotted in Fig. 3(b). The error bars cover their variation with the $q$ 's, expected because the sampled timescale $\tau(q)$ of the system would also select a $\operatorname{CRR}(q)$ by virtue of the spatio-temporal coupling. ${ }^{29)}$ The CRR subsystem treatment is justified here, ${ }^{28)}$ as $N \geq 35$ for all the $x$ 's shown. Typical $N=35$ and $\zeta=\sqrt[3]{3 V / 4 \pi}=0.63 \mathrm{~nm}$ of CRRs in $\mathrm{Ge}_{19} \mathrm{Se}_{81}$ closely resemble those of the Crown glass.

To understand the $V_{\mathrm{CRR}}(x)$ as obtained and reconcile it with the $E_{\mathrm{a}}(x)$, we examine the structural processes underway. The stiffness development in $\mathrm{Ge}_{x} \mathrm{Se}_{1-x}$ (with increasing Ge doping) proceeds through the recession of soft (long \& uniform Se-chains) matrix giving way to rigid clusters whose internal configuration starts out as corner-shared $\mathrm{Ge}\left(\mathrm{Se}_{1 / 2}\right)_{4}$ tetrahedra. ${ }^{30)}$ These ring-clusters grow up to a certain size and get interconnected via short $(\mathrm{Se})_{\mathrm{m}}$ links as $x_{\mathrm{p}} \approx 0.2$ is approached, forming a quasi-global network. ${ }^{31)}$ The result is a relatively compact space filling, with trapped isolated soft pockets. From activation energy and the CRR size, the evolving range $0.15 \leq x<0.2$ appears as an anomalous phase and the exact $x_{\mathrm{p}}$ composition seems to be a regularly disordered one-a.k.a. continuous random network (CRN) having defects. The extremal behaviour of $E_{\mathrm{a}}$ and $V_{\mathrm{CRR}}$ for lower $x$ 's indicates an excursion riding over the expected gradual development of structural networking in the system.

Further increase in Ge concentration $\left(x>x_{\mathrm{p}}\right)$ requires a metamorphosis of the inter-tetrahedral configuration to the edge-shared kind, thereby receding the optimized packing. This simultaneously erodes the connectivity of a structurally robust $\mathrm{GeSe}_{4}$, making way for another, somewhat softer material-phase, believed to have the backbone-form ${ }^{32,33)}$ of random necklaces with varying-sized blob-pearls comprising of edge-shared $\mathrm{Ge}\left(\mathrm{Se}_{1 / 2}\right)_{4}$. For $x>x_{\mathrm{p}}$, though no significant changes are observed in $E_{\mathrm{a}}$, indicating cessation of the kinetic evolution, the structural de-optimization manifests in the cooperative dynamics as indicating another distinct phase. This may well be the recently predicted intermediate phase, based on theoretical treatment of CRN-variants by Thorpe and Phillips, ${ }^{34)}$ taking into account the fluctuations from a mean-field behaviour, and probably manifested in recent high-resolution Raman experiments. ${ }^{35}$ )

The competition between attainment of a rigidly connected quasi-3D network and its changeover into relatively fragile quasi-2D network seems to endow the $x_{\mathrm{p}}=0.2$ composition with a "saddle point" character, that conforms most to the Arrhenicity as regards its thermokinetics. Moreover, the interplay also irons out the irregular drive-response profile observed on either side of $x_{\mathrm{p}}$, resulting in the linearity of heatflow-shift at $T_{\mathrm{g}}$ with respect to the heating ramp for $\mathrm{GeSe}_{4}$, standing out as the 'inflexion' composition. Technically, the linearity of $\Delta H(q)$, highlighted for $\mathrm{GeSe}_{4}$ corresponds to its 
coupling parameter $\Delta C_{\mathrm{p}}(=-\Delta H / q)$ being independent of the driving temperature ramp-rate. In addition, the present work also opens up the question of whether and how the deviations from linearity in the kinematics (drive vs. response) and kinetics (system vs. probing time) behaviour is related.

\section{Conclusion}

Glass transition kinetics studies are reported near the mechanical threshold $x_{\mathrm{p}}=0.2$ of $\mathrm{Ge}_{x} \mathrm{Se}_{1-x}$ series, where a rigidity phase transition has been evident. Anomalies observed in the response-linearity, thermal activation energy, and dynamical heterogeneity are traced to two distinct phases ascribed to the composition-ranges below and above $x_{\mathrm{p}}$, by correlating with the underlying structural attributes. The results are important in applying theoretical tools to rigidity percolation in this system, and in selecting the optimal composition for applications.

\section{Acknowledgements}

SS acknowledges CSIR for the research fellowship no. 9/681(4)/97-EMR-I.

\section{REFERENCES}

1) R. Zallen: The Physics of Amorphous Solids, (John Wiley \& Sons, 1983).

2) J. C. Phillips: J. Non-Cryst. Solids 34 (1979) 153.

3) J. C. Phillips: J. Non-Cryst. Solids 43 (1981) 37.

4) M. F. Thorpe: J. Non-Cryst. Solids 57 (1983) 355.

5) C. A. Angell: J. Non-Cryst. Solids 131-133 (1991) 13.

6) G. H. Dohler, R. Dandoloff and H. Bilz: J. Non-Cryst. Solids 42 (1980) 87.

7) T. Wagner, S. O. Kasap, M. Vlcek, A. Sklenar and A. Stronski: J. NonCryst. Solids 227 (1998) 752.

8) I. Alig, W. Jenninger and J. E. K. Schawe: J. Non-Cryst. Solids 235-237 (1998) 504

9) D. Tonchev and S. O. Kasap: J. Non-Cryst. Solids 248 (1999) 28 and references cited therein.

10) U. Senapti, K. Firstenberg and A. K. Varsheneya: J. Non-Cryst. Solids 222 (1997) 153 and references cited therein.

11) C. T. Moynihan, S. N. Crichton and J. M. Opalka: J. Non-Cryst. Solids 131-133 (1991) 420.

12) G. Adams and J. H. Gibbs: J. Chem. Phys. 28 (1965) 373.

13) R. Azoulay, H. Thibirge and A. Brenac: J. Non-Cryst. Solids 18 (1975) 33.

14) F. Xingwei, W. J. Bresser and P. Boolchand: Phys. Rev. Lett., 78 (1997) 4422.

15) A. Feltz, H. Aust and A. Blayer: J. Non-Cryst. Solids 55 (1983) 179.

16) Y. Wang, O. Matsuda, K. Inoue, O. Yamamuro, T. Matsuo and K. Murase: J. Non-Cryst. Solids, 232-234 (1997) 702

17) J. E. K Schawe and S. Theobald: J. Non-Cryst. Solids 235-237 (1975) 496 and refs. therein.

18) Disorder Effects in Relaxational Processes, Eds. R. Richert and A. Blumen (Springer-Verlag, 1994) 23.

19) C. A. Angell: J. Phys. Chem. Solids, 49 (1988) 863.

20) M. H. Cohen and D. Turnbull: J. Chem. Phys, 31 (1959) 1164.

21) Disorder Effects in Relaxational Processes, Eds. R. Richert and A. Blumen (Springer-Verlag, 1994) 75.

22) G. P. Johari and M. Goldstein: J. Chem. Phys, 53 (1970) 2372 and references cited therein.

23) H. Vogel: Z. Phys., 22 (1921) 645

24) G. S. Fulcher: J. Am. Ceram. Soc., 8 (1925) 339

25) H. Sillescu: J. Non-Cryst. Solids 243 (1999) 81 and references cited therein.

26) P. Schellenberg and J. Friedrich: Disorder Effects in Relaxational Processes, Eds. R. Richert and A. Blumen (Springer-Verlag, 1994) 407.

27) H. W. Spiess and K. Schmidt-Rohr: Disorder Effects in Relaxational Processes, Eds. R. Richert and A. Blumen (Springer-Verlag, 1994) 385.

28) E. Donth: J. Non-Cryst. Solids 53 (1982) 325 and references cited therein.

29) E. Donth: J. Non-Cryst. Solids 131-133 (1991) 204.

30) M. Cobb, D. A. Darbold and R. L. Cappelletti: Phys. Rev. B, 54 (1996) 12162.

31) N. Ramesh Rao, P. S. R. Krishna, S. Basu, B. A. Dasannacharya, K. S. Sangunni and E. S. R. Gopal: J. Non-Cryst. Solids 240 (1998) 221 and references cited therein.

32) L. F. Gladden and S. R. Ellliot: Phys. Rev. Lett. 59 (1987) 908.

33) H. J. Hertmann and H. E. Stanley: Phys. Rev. Lett., 53 (1984) 1121.

34) M. F. Thorpe, D. J. Jacobs, M. V. Chubynsky and J. C. Phillips:J. NonCryst. Solids 266-269 (2000) 859.

35) D. Selvanathan, W. J. Bresser and P. Boolchand: Phys. Rev. B, 61 (2000) 15061. 